\title{
The ATNF Pulsar Catalog
}

\author{
G. Hobbs, R. Manchester, A. Teoh, M. Hobbs \\ Australia Telescope National Facility - CSIRO, P.O. Box 76, Epping \\ NSW 1710, Australia
}

\begin{abstract}
The number of known pulsars has significantly increased over the past few years. We have searched the literature to find papers announcing the discovery of pulsars or giving improved parameters for them. Data from the papers have been entered into a new pulsar catalog that can be accessed via a web interface or from the command line (on Solaris or Linux machines). The user may request over 120 different parameters, select pulsars of interest, generate custom variables and choose between different ways of displaying or tabulating the data. Full bibliographic references are available for all observed parameters.
\end{abstract}

\section{Introduction}

We have produced a new catalog (accessed via a web or command-line interface) that contains all published rotation-powered pulsars, including those detected only at high energies. The catalog also contains anomalous X-ray pulsars (AXPs) and soft gamma-ray repeaters (SGRs) for which coherent pulsations have been detected. We have excluded accretion-powered pulsars such as Her X-1 and the recently discovered X-ray millisecond pulsars (e.g. SAX J1808.4-3658; Wijnands \& van der Klis 1998). We currently store information on 1300 pulsars.

For each pulsar we record up to 90 different parameters with full bibliographic information. For all pulsars we store names, positions, rotational frequencies and the type of pulsar (e.g. AXP). If published values exist, we also store parameters such as higher-order rotational frequency derivatives, pulse widths, orbital parameters, proper motions and any associations with globular clusters, the Magellanic clouds or supernova remnants. If enough information exists to calculate derived parameters then these may also be displayed using the web or command-line interfaces. Currently, 39 different derived parameters are included.

\section{The Web Interface}

A web interface (http://www.atnf.csiro.au/research/pulsar/psrcat) has been developed to allow easy global access to our pulsar catalog. The user may select parameters of interest, generate custom variables, select wanted (or unwanted) pulsars and tabulate the results in a variety of different forms. For browsers with Java enabled, the results may also be displayed graphically, as a two dimensional plot or as a histogram, using an interactive applet (see Fig. 1). We 


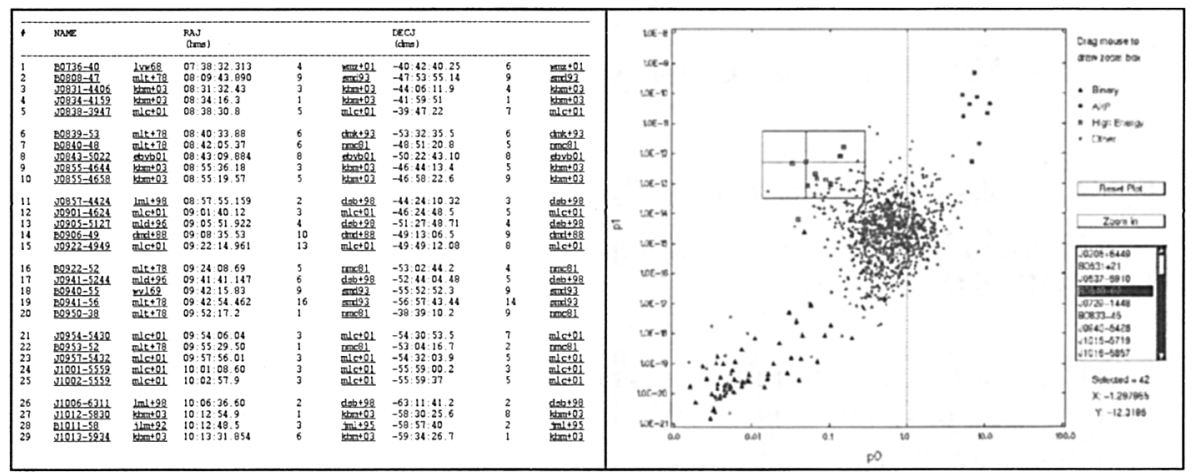

Figure 1. An example of the "long-form" of tabular output and the graphical plotting interface.

also provide a special ("expert") mode of operation that can be accessed from http://www.atnf.csiro.au/research/pulsar/psrcat/expert.html. This allows the user to obtain pulsar parameters of specialist interest and to upload one or more personal catalogs to use instead of, or to merge with, the public catalog. Such merging is not permanent and does not affect any other user of the system.

\section{The Command-line Interface}

Data can be obtained from the public or from a personal catalog using a command line interface. This software has been tested on both Solaris and Linux machines and can be obtained via ATNF anonymous ftp (see authors for details). This software provides the same functionality as the web-based interface with the exception of the plotting routines. C functions are available to access the catalog data directly.

Acknowledgments. Many people have contributed to the maintenance and upgrading of earlier versions of the database. We particularly thank Andrew Lyne of the University of Manchester, David Nice of Princeton University and Russell Edwards, then at Swinburne University of Technology. This work has made extensive use of NASA's Astrophysics Data System and the SLALIB $\mathrm{C}$ routines.

\section{References}

Wijnands, R., \& van der Klis, M. 1998, Nature, 394, 344 